\title{
COMPTES RENDUS BIBLIOGRAPHIQUES
}

La Revue Archéologique donne, dans chacun de ses numéros, la liste des ouvrages reçus, mais elle ne peut s'engager à rendre compte que des ouvrages qu'elle aurait elle-même demandés.

Aubrey Pomerance \& Bettina Schmitz (éd.), Heiligtümer, Papyri und geflügelte Göttinnen. Der Archäologe Otto Rubensohn (Ḧ̈B 53), Hildesheim, 2015, in-4º, 120 p., ISBN 978-3-8067-8806-8.

C'est à l'occasion de la donation en 2006 au Musée Juif de Berlin, par Fortunatus Schnyder-Rubensohn, des archives de son beau-père, l'archéologue Otto Rubensohn (1867-1964) et de l'inauguration des salles où elles sont présentées, qu'y fut organisé, le 18 février 2010, un symposium retraçant la carrière du savant disparu. Le nom donné à ces salles est le même que celui du volume qui en contient les actes: "Sanctuaires, papyri et déesses ailées : l'archéologue Otto Rubensohn ". À travers six communications, et après un mot d'accueil de Thomas Schnyder, son petit-fils, sont décrits la carrière et les travaux de l'archéologue allemand.

Aubrey Pomerance, "Otto Rubensohn. Eine biographische Skizze » (p. 12-25), rappelle le parcours, la formation et le destin de l'archéologue qui mourut à Bâle trois mois avant son $97^{\mathrm{e}}$ anniversaire, le 9 août 1964. C'est à Cassel, capitale de l'ancien électorat de Hesse qui venait d'être annexé par la Prusse, que le 23 novembre 1867 naît Otto Rubensohn, c'est là qu'il acquiert une formation en philologie classique, avant de partir, en 1887, à l'Université de Berlin pour y poursuivre ses études en philologie classique, en archéologie et en histoire de l'art. En 1888, il poursuit son cursus à la Kaiser-Wilhelms-Universität de Strasbourg, où il soutient une thèse de doctorat en 1892, préparée sous la direction d'Adolf Michaelis : Die Mysterienheiligtümer in Eleusis und Samothrake (Berlin, 1892). Après avoir obtenu les certificats d'aptitude nécessaires pour enseigner dans le secondaire le latin, le grec, l'histoire et l'allemand, et après avoir été libéré de ses obligations militaires, il est enseignant dans un Lycée de Potsdam. Tout au long de sa carrière, il alternera les périodes de recherches académiques avec celles de l'enseignement dans différents lycées (Gymnasien).
C'est finalement à l'automne 1897 qu'il obtient un premier engagement, comme archéologue, auprès de l'Institut archéologique allemand d'Athènes, placé sous la direction de Wilhelm Dörpfeld (1853-1940) : il fouille sous l'Acropole d'Athènes, à Éleusis et à Céphisie. De 1898 à 1899, il va diriger des fouilles à Paros. Toutefois, dès novembre 1898 il reçoit des Musées royaux de Berlin la proposition de diriger des fouilles à Abousir en Égypte. Après les campagnes de 1899 en Égypte et en Grèce, il rentre en Allemagne et retourne dans l'enseignement secondaire. Dès 1901, il obtient un nouveau contrat du «Papyrus-Kartell» formé autour des Musées royaux de Berlin pour acquérir en Égypte des papyri et faire des fouilles là où on était susceptible d'en trouver : dans le Fayoum (1902), à el-Achmounein (1903-1906), à Abousir el-Méleq (1902-1905) et enfin à Éléphantine (1906-1907). À partir de 1905, Otto Rubensohn essaie en vain d'obtenir un emploi permanent auprès des Musées royaux de Berlin, aussi finit-il par retourner enseigner au Gymnasium. Il épouse en 1909 Frieda Oppler et de leur union naît en 1914 une fille, Käte. De 1909 à 1915, il est le directeur de la Pelizaeus-Sammlung qui venait d'être fondée (1907) au sein du Musée Roemer d'Hildesheim et obtient le 2 juillet 1910 le titre de Professor. Puis, de 1915 jusqu'à sa pension en 1932, il enseigne de nouveau dans divers Gymnasien en qualité de Staatlicher Oberlehrer. Le moment de la retraite devait être pour lui l'occasion de publier les résultats de ses travaux archéologiques que ses années d'enseignement dans le secondaire n'avaient pu lui permettre de mettre au point. Toutefois, la prise du pouvoir et la mise en place de la dictature nationale-socialiste, et les persécutions qui s'en suivirent, l'isolèrent dramatiquement du monde académique : 
en 1938 (il a 71 ans !), la Deutsche Orient-Gesellschaft et la Vereinigung der Freunde antiker Kunst le congédient brutalement et l'accès aux musées et aux bibliothèques lui est désormais interdit. À l'issue des pogromes de novembre 1938, sa fille Käte organise l'émigration de ses parents vers la Suisse où elle étudie: en mars 1939, Otto Rubensohn s'installe à Bâle et s'il a été en mesure d'emporter avec lui sa collection d'antiquités, la famille est privée de ressources et bénéficie surtout à ce moment-là du soutien financier de Mimi Borchardt (1877-1948), veuve de son ami de longue date, l'égyptologue Ludwig Borchardt (1863-1938). Enfin, c'est grâce à l'aide de ses collègues bâlois, l'archéologue Karl Schefold (1905-1999) et l'historien de l'Antiquité Felix Staehelin (1873-1952), qu'il peut reprendre ses travaux scientifiques: article de synthèse sur Paros pour la Realencyklopädie de Pauly-Wissowa, un article sur la déesse ailée de Délos qui paraîtra en 1948 dans le premier volume des Mitteilungen des Deutschen Archäologischen Instituts, Athenische Abteilung publié après guerre et enfin, en 1962, à l'âge 95 ans, il fait paraître son ouvrage Das Delion von Paros (Wiesbaden, Franz Steiner Verlag), c'est cette même année, pour son anniversaire, que le Président de la République Fédérale d'Allemagne, K. Heinrich Lübke (1894-1972), lui décerne la Croix du Mérite $1^{\text {re }}$ classe; il décède deux ans plus tard.

L'article de Vasiliki Barlou, «'... Am Ort meiner Bestimmung'. Otto Rubensohns Forschungen auf Paros 1898-1899" (p. 26-39), expose les travaux archéologiques et les recherches d'O. Rubensohn sur l'île de Paros. C'est surtout en différents endroits de la localité principale, Parikia, qu'il commence par conduire des fouilles: fondations du temple archaïque d'Athéna, recherche de l'endroit où s'élevait le sanctuaire de Déméter Thesmophoros mentionné par Hérodote (VI, 134), recensement des éléments antiques remployés dans la forteresse vénitienne de Parikia (Kastro). Mais les travaux majeurs qu'il entreprit à cette époque concernaient le sanctuaire d'Asclépios du Iv ${ }^{\mathrm{e}} \mathrm{s}$. av. J.-C. : il y découvrit notamment, en 1898, un remarquable kouros de taille humaine, aujourd'hui conservé au musée du Louvre (Ma 3101). En plus de la conduite des fouilles, O. Rubensohn participa à la fondation du premier musée de Paros. Lors de sa seconde et dernière campagne de trois mois en 1899 , il poursuivit ses fouilles en divers endroits, dans l'Asklépiéion, mais aussi autour du Kastro où il mit au jour des structures très anciennes, datables en 2000/1600 av. J.-C. Il fouilla aussi une nécropole d'époque hellénistique et romaine. Mais le couronnement de ses travaux à Paros est la découverte du Délion, c'est-à-dire le sanctuaire d'Apollon, situé à l'endroit le plus élevé des montagnes au nord de l'entrée de la baie de Paros. Celui-ci ne sera finalement publié par son inventeur que 63 ans plus tard.

Josefine Kuckertz, "Auf der Jagd nach Papyri. Otto Rubensohn in Ägypten " (p. 40-59), traite des activités d'O. Rubensohn en Égypte, celles conduite à Abousir en 1898/1899 pour le compte des Musée de Berlin mais aussi celles entreprises entre 1901 et 1907 dans le cadre du « Papyrus-Kartell ».J. Kuckertz précise en quoi consistait ce Papyrus-Kartell fondé autour des Musées royaux de Berlin : il s'agissait de l'association de plusieurs institutions allemandes intéressées par l'acquisition de papyri grecs, qui mutualisèrent ainsi leurs moyens pour acheter des papyri dans le commerce des antiquités et pour financer des fouilles susceptibles d'en découvrir d'autres ; placé sous la direction de l'administration des Musées Royaux de Berlin, il associait en outre Leipzig, Gießen, Würzburg et Strasbourg, auxquels par la suite se joindront d'autres institutions académiques. Le "Papyrus-Kartell » cessa de fonctionner en 1912. O. Rubensohn arriva en Égypte en 1898 pour conduire les fouilles du complexe solaire de Niouserrê à Abousir, dont les musées berlinois venaient d'acquérir un certain nombre de reliefs. Les fouilles se déroulèrent pendant l'hiver 1898/1899, puis se poursuivirent, sans O. Rubensohn, en $1899 / 1900$ et 1900/1901 avec le financement et sous la conduite du baron Friedrich Wilhelm von Bissing (1873-1956), puis jusqu'en 1908 dans le cadre de la Deutsche Orient-Gesellschaft, sous la direction de L. Borchardt. Entre 1901-1907, O. Rubensohn est engagé pour le compte du "Papyrus-Kartell" dans la chasse aux papyri. Au-delà de la gestion des acquisitions auprès des marchands cairotes et des expéditions d'antiquités, il s'agissait d'entreprendre des fouilles. D'abord dans le Fayoum en 1901/1902, à Théadelphie (Batn Harit) en février 1902, puis en mars de la même année à Abou Hamid, enfin à partir du 23 mars, et pour deux semaines, à Tebtynis (Umm el-Bareigat) ; un site que les anglais B. P. Grenfell (1841-1925) et A. S. Hunt (1871-1934) avaient déjà fouillé en 1899/1900, avec d'importantes trouvailles. O. Rubensohn fouille des maisons et 
la nécropole de crocodiles sacrés d'où il emporte à Berlin des momies de crocodiles : les cartonnages de celles-ci étant généralement constitués de papyri réutilisés. Dès le mois d'avril, $\mathrm{O}$. Rubensohn et son équipe (150 ouvriers!) gagnent la Vallée, et plus précisément Abousir el-Méleq, une des nécropoles d'Hérakléopolis Magna. Il y fouillera régulièrement jusqu'en 1905 et, au cours de sa troisième campagne, en 1904, il met au jour la tombe intacte d'une certaine Tadja, contemporaine de la $\mathrm{xxv}^{\mathrm{e}}$ dynastie et de nombreux papyri. De 1903 à 1906, il conduit des fouilles à el-Achmounein, la nécropole d'Hermopolis Magna. De 1906 à 1907, c'est Éléphantine qui constitue son grand chantier, puisqu'il découvrit dans l'agglomération située autour du temple de Khnoum de nombreux papyri d'époques variées, hiératiques, démotiques, araméens et grecs, dont beaucoup sont aujourd'hui conservés au Neues Museum de Berlin.

Dans son article «Otto Rubensohn Ausgrabungen in Abusir el-Meleq. Bermerkungen zu einigen herausragenden Funden" (p. 60-73), Klaus Parlasca revient plus en détail sur certaines découvertes faites à Abousir el-Méleq, plus particulièrement les fouilles des secteurs de l'époque tardive à l'époque romaine. Les archives du fouilleur, déposées au Musée Juif de Berlin, permettent désormais de préciser un certain nombre de données sur ces travaux. Ainsi, au sujet de la sépulture collective des prêtres d'Hérichef, du matériel qu'elle contenait et de sa dispersion à travers les musées on en sait un peu plus désormais. Kl. Parlasca décrit le matériel funéraire tardif mis au jour au cours de ces fouilles et ses spécificités : Sarcophage-chasse, portraits de momies, cartonnages. Il évoque l'extraction des papyri des cartonnages dont ils sont constitués. Enfin, il replace ce matériel d'Abousir el-Méleq dans le contexte général des traditions funéraires de l'époque ptolémaïque et romaine.

Bettina Schmitz, "Otto Rubensohns Hildesheimer Jahre, 1909-1915, Gründungsdirektor des Pelizaeus-Museums " (p. 74-87), évoque les six années (1909-1915) que passa Otto Rubensohn en qualité de directeur-fondateur du Pelizaeus-Museum au sein du Roemer-Museum d'Hildesheim. Si le Roemer-Museum existe depuis 1844, c'est en 1907 qu'est créée en son sein la Pelizaeus Sammlung, par la volonté de Wilhelm Pelizaeus (1851-1930), riche marchand d'import-export, banquier et consul, installé en Égypte de 1869 à 1914, qui souhaitait offrir à sa ville natale, Hildesheim, la très importante collection qu'il avait constituée. C'est en 1906 qu'Otto Rubensohn, qu'il avait connu au Caire dès 1901, et qui lui avait été recommandé par Adolf Erman (1854-1937) et Ludwig Borchardt, devint son conseiller scientifique. Le $1^{\text {er }}$ avril 1909, O. Rubensohn est engagé comme directeur en second du Musée Roemer (qui deviendra par la suite le Musée Roemer et Pelizaeus), et prend en charge le déménagement de la collection depuis l'Égypte jusqu'aux bords de l'Innerste, son installation et sa présentation dans l'ancien orphelinat luthérien qui devait l'abriter. La collection d'antiquités égyptiennes et gréco-romaines comptait pas moins de 2000 objets au moment de son ouverture. Otto Rubensohn devait aussi surmonter toutes sortes de difficultés, notamment les retards pris par les travaux de préparation du bâtiment, mais la collection ouvrit finalement ses portes au public le 29 juillet 1911. En 1912, O. Rubensohn est titularisé comme directeur de la collection; il démissionne pourtant en 1915, pour des raisons que B. Schmitz essaie d'éclairer à partir des rares archives disponibles. O. Rubensohn aurait été compromis dans une " affaire » interne au musée : il aurait entretenu une liaison avec une secrétaire de l'institution, ce qui n'était guère admissible dans la prude Allemagne provinciale de l'époque. À l'été 1915, il s'installe avec sa famille à Berlin et s'en retourne enseigner dans le secondaire, jusqu'à son départ à la retraite en 1932 , à 65 ans.

Le dernier article de l'ouvrage, celui de HeinzHelge Nieswandt et Dieter Salzmann, "Ausgewählte Artefakte der Sammlung Rubensohn » (p. 88-107), est une présentation générale de la collection privée d'antiquités grecques, égyptiennes et chypriotes constituée par Otto Rubensohn. La collection de l'archéologue a été acquise, après sa mort, par l'intermédiaire d'un de ses anciens élèves, le $D^{r}$ Ernst H. Vits (1903-1970), par le Musée archéologique de l'Université de Münster, dont les collections avaient été détruites au cours de la Seconde Guerre mondiale. Huit objets sont présentés de manière détaillée : une petite tête de Sérapis ; une statuette d'Aphrodite; un fragment de figurine en terre cuite représentant un Gaulois; un louterion miniature en argile; un petit autel en calcaire ; une petite coupe attique à figure noire; une lampe à huile et, finalement, une anse de lampe figurative représentant un singe lecteur. 
Une bibliographie d'Otto Rubensohn conclut l'ouvrage.

La publication des actes de ce symposium est bienvenue, elle offre de nombreuses informations nouvelles sur la carrière et l'œuvre d'Otto Rubensohn. Le savant fouilla des sites importants et la documentation considérable qu'il mit au jour continue à faire l'objet de nombreux travaux et on peut espérer que l'exploitation de ses archives, généreusement offertes par ses descendants au Musée
Juif de Berlin, permettra de préciser de nombreux points sur les circonstances de leur découverte. Le seul bémol que l'on peut exprimer au sujet de cette publication est la médiocre qualité de la reproduction des illustrations.

Ivan GUERMEUR, CNRS (UMR 5140, ASM, Montpellier). Ivan.GUERMEUR@cnrs.fr

BALANDiER Claire (éd.), Nea Paphos, Fondation et développement urbanistique d'une ville chypriote de l'Antiquité à nos jours. Études archéologiques, historiques et patrimo-

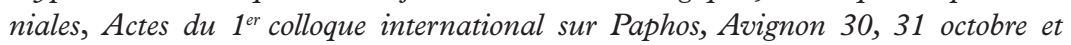

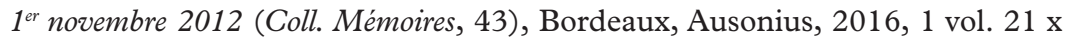
$29,7,438$ p., fig. ds t.

L’ouvrage dirigé par Claire Balandier constitue la publication d'un colloque international tenu à l'automne 2012 en Avignon, dont l'ambition était d'offrir une synthèse des études récentes sur la ville chypriote de Nea Paphos. On doit souligner d'emblée l'excellente facture du volume, proprement édité et qui fait la part belle aux illustrations, ce qui ravira les lecteurs sensibles à la documentation graphique et désireux d'y trouver en un seul endroit des plans, des relevés architecturaux et des photographies de bonne qualité. L'utilisation de la couleur - qui n'élève pas excessivement le prix de l'ouvrage - est le plus souvent judicieuse, même si l'on peut formuler un regret en ce qui concerne certains tableaux statistiques parfois illisibles (par ex. fig. 3, p. 190). Il est préjudiciable, d'autre part, de ne trouver nulle part un plan qui détaille les fouilles menées à Nea Paphos par les différentes missions, chypriotes et étrangères, ni même une présentation d'ensemble des vestiges antiques et médiévaux mis au jour. Ainsi, seul un lecteur averti peut aisément se promener dans la topographie parfois complexe de la ville. Ce livre se distingue également par le soin apporté à l'édition ainsi que par le très faible nombre de coquilles et de fautes, bien que les articles soient rédigés dans trois des principales langues de la recherche scientifique à Chypre (italien, français et anglais). Il est inutile de s'attarder sur les petites erreurs qui parsèment çà et là l'ouvrage : c'est un fait inhérent à toutes les publications du genre, même si l'éditrice des actes regrettera sans doute de voir curieusement son nom devenir Claire Besson (sic) en couverture intérieure.

L'un des grands mérites du volume est intrinsèquement lié au champ d'étude qu'il couvre, volontairement large, embrassant une chronologie longue, qui a conduit à faire intervenir les plus grands spécialistes de la recherche paphienne. Les trente-deux contributions sont divisées en trois parties : les deux premières présentent successivement " la recherche archéologique à Paphos " (p. 33-229), puis les "approches historiques de Nea Paphos" (p. 233-369), où se côtoient habilement des synthèses pertinentes et des articles plus restreints qui offrent de nouveaux points de vue sur des sujets précis (article de J.-B. Cayla notamment, p. 275-285). Cette division est tout à fait acceptable, même si nous étions en droit d'attendre un regroupement des diverses contributions sur la numismatique et l'histoire monétaire, présentées dans l'une ou l'autre des grandes parties (art. d'A. Destrooper-Georgiadès, p. 177-188, d’É. Markou, p. 233-240 et de J. Olivier et P. Keen, p. 261-274).

La troisième partie du volume, naturellement plus courte, est intitulée "le patrimoine à l'épreuve des siècles » (p. 375-433): c'est une initiative rare dans les colloques consacrés à l'histoire et à l'archéologie antiques et médiévales. Elle est ici bienvenue, car elle met en lumière la problématique indispensable 